\title{
Patient Experiences with Chronic Care Management Services and Fees: a Qualitative Study
}

\author{
Claire Wilson, $\mathrm{PhD}^{7}$, Ann S. O'Malley, MD, MPH' , Carla Bozzolo, $\mathrm{MPH}^{7}$, Nancy McCall, $S c D^{2}$, and \\ Sai Ma, $P h D^{3}$ \\ ${ }^{1}$ Insight Policy Research, Arlington, VA, USA; ${ }^{2}$ Mathematica Policy Research, Washington DC, USA; ${ }^{3}$ Centers for Medicare \& Medicaid Services, \\ Baltimore, MD, USA.
}

\begin{abstract}
BACKGROUND: As of 2015, the Centers for Medicare \& Medicaid Services (CMS) pays for chronic care management (CCM) services for Medicare beneficiaries with two or more chronic conditions. CMS requires eligible providers to first obtain patients' verbal (and, prior to 2017, written) consent, to ensure that patients who participate in CCM services understand their rights and agree to any applicable cost sharing. CCM providers must also enhance patients' access to continuous and coordinated care, including ongoing care management.
\end{abstract}

OBJECTIVE: To understand patients' perceptions of the consent process, their reasons for choosing to participate, and their experiences receiving $\mathrm{CCM}$ services.

DESIGN: Qualitative study using semi-structured interviews with Medicare beneficiaries who had two or more CCM claims submitted by an eligible provider. Beneficiaries were selected from a sampling frame of Medicare claims submitted between January and September 2015.

KEY RESULTS: Most patients reported no concerns about being asked to participate in CCM. The majority of patients had secondary insurance (or Medicaid) that covered any coinsurance for CCM and therefore could not say with certainty whether they would participate if they had to pay for CCM services out-of-pocket. Reasons for participating included having insurance that covered the copay and peace of mind about having access to the CCM team. Patients reported multiple benefits of participating in CCM services, including better access to their primary care team, improved continuity of care, and improved care coordination. Most patients reported no downside to participating in CCM services, although some felt they were relatively healthy and questioned whether they needed CCM services.

CONCLUSIONS: These findings on patients' experiences participating in CCM services during the first 9 months of the policy's implementation can help providers and policymakers understand their perceived benefits and unintended consequences. Our findings also have implications for providers when approaching patients about CCM services.

Electronic supplementary material The online version of this article (https://doi.org/10.1007/s11606-018-4750-x) contains supplementary material, which is available to authorized users.

Received January 18, 2018

Revised July 10, 2018

Accepted November 2, 2018

Published online December 3, 2018
KEY WORDS: care management; chronic conditions; patient experiences; qualitative research; care coordination.

$\mathrm{J}$ Gen Intern Med 34(2):250-5

DOI: $10.1007 / \mathrm{s} 11606-018-4750-\mathrm{x}$

(C) Society of General Internal Medicine 2018

\section{INTRODUCTION}

The rising prevalence of chronic disease represents one of the most significant challenges facing the US healthcare system. ${ }^{1}$ Two thirds of Medicare beneficiaries have two or more chronic health conditions. ${ }^{2}$ Medicare beneficiaries with multiple chronic conditions have more hospitalizations, physician visits, and emergency room visits compared to beneficiaries with one or no chronic conditions, and they account for $93 \%$ of Medicare spending. ${ }^{2}$ Though they receive services from multiple specialists, these patients rely first and foremost on primary care providers for their healthcare needs. ${ }^{3}$ Primary care practices, however, often receive inadequate financial and infrastructure support to provide the care coordination and management that these patients require. ${ }^{4}$ Compounding this problem, high rates of patient self-referral to specialists and specialist-to-specialist cross referrals lead to fragmented care, which is associated with increased healthcare costs, reduced quality of care, ${ }^{5,6}$ and increased coordination burden for primary care practices.

A growing body of research suggests comprehensive care management and coordination of services can help improve health outcomes and reduce costs for patients with multiple chronic conditions. $^{7-9}$ In January 2015, the Centers for Medicare \& Medicaid Services (CMS) implemented a new policy to pay providers for chronic care management (CCM) services delivered to Medicare beneficiaries with two or more chronic conditions, ${ }^{10}$ with the goal of improving health and quality of care for these high-need patients. Eligible CCM providers, including physicians, nurse practitioners and physician assistants, clinical nurse specialists, and nurse midwives, may now bill CMS for up to $20 \mathrm{~min}$ of non-face-to-face care management services per patient in a 30-day period. The rate for standard CCM (CPT Code 99490) is $\$ 43$, and patients are responsible for a $20 \%$ coinsurance payment on the billable services, which amounts to approximately $\$ 8$ for the patient, 
though this fee is typically covered for patients with a Medigap policy or dual enrollment in Medicare and Medicaid. A Medigap policy is a supplemental health insurance plan sold by private insurance companies to cover costs not covered under original Medicare. In some cases, a patient's $20 \%$ might be greater than $\$ 8$, if the practitioner works in a setting that can charge a facility fee.

CCM includes an extensive scope of services, such as ensuring continuity of care with a designated clinician; developing and revising a comprehensive care plan in collaboration with the beneficiary; communicating with other health professionals, including hospitalists, specialists, and other providers; $24 / 7$ access to healthcare professionals to address urgent needs; and ongoing medication management. Practices must also have a certified electronic health record and ensure that the care plan is available electronically at all times to anyone providing CCM services.

CMS also requires CCM providers to initiate a discussion with eligible patients about CCM services and associated fees and obtain the patient's written or verbal consent before billing for the services. The initial discussion is intended to ensure patients understand what CCM services include, how health information will be shared with other providers caring for the patient, how cost sharing applies to these services, and how patients can revoke the services or choose a different eligible provider as their $\mathrm{CCM}$ provider. Though there are no requirements that providers use a standard script or consent form when requesting patient consent, some professional associations have developed sample introductory letters and templates that practices can use with eligible patients.

To our knowledge, nothing has been published to date on Medicare beneficiaries' experiences with the new CCM services and fee. To better understand patients' experiences and perceptions of CCM, we interviewed 45 Medicare beneficiaries and 3 caregivers of beneficiaries for whom providers had billed for CCM services within the first 9 months of the new policy. As more providers consider CCM services for patients with multiple chronic conditions, it will be important to understand the perceived benefits and unintended consequences that patients report.

\section{METHODS}

We developed a semi-structured interview protocol to gather information about patients', or their caregivers', perceptions of CCM (see online Appendix).

The semi-structured interview protocol addressed five topics:

- How patients learn about CCM services and their reported first impressions

- Patient perceptions of the informed consent process

- Role of supplemental insurance in beneficiary decisionmaking

- Reasons for providing consent
- Care continuity and communication with CCM practice

- Timely access to care

- Care management and care transitions

Pilot testing of the interview protocol among three beneficiaries resulted in minor changes to improve flow. The language in the screener was also updated after a few interviews to more closely reflect the way patients think about CCM services.

\section{Recruitment and Data Collection}

This study is exempt (45 CFR 46.101[b]) from institutional review board (IRB) review because it was conducted subject to the approval of the head of CMS and was designed to evaluate the CCM services program.

We created the sampling frame for the beneficiary interviews using Medicare Part B and outpatient claims from January to September 2015. Medicare Part B is the federal health insurance program that covers preventive services, outpatient care, and medical supplies for eligible beneficiaries, including people age 65 or over, under 65 with disabilities, or with end-stage renal disease. We identified all beneficiaries who had two or more CCM claims submitted by an eligible provider. We selected a random sample of 500 beneficiaries from the sample frame, stratified by age, state, provider type, and type of coverage (e.g., Medicare, Medicare plus supplemental insurance, Medicare-Medicaid). The characteristics of participating beneficiaries were similar to Medicare beneficiaries who received CCM services nationally. Compared to the general Medicare fee-for-service (FFS) population, CCM beneficiaries tend to be sicker, they are more likely to be in smaller practices, and they are more likely to reside in the South. ${ }^{11}$

Between February and April 2016, interviewers sent an advance letter to beneficiaries on a rolling basis, via express mail, and followed up with each recipient by phone within a few days of the mailing. The advance letter explained the study, requested the beneficiary's participation, and stated that beneficiaries would receive $\$ 25$ as appreciation for their participation. We over-selected beneficiaries from four states with the most Medicare beneficiaries (California, Florida, Texas, and New York) and aimed to get a geographically diverse selection of beneficiaries from other states.

A team of four interviewers screened each beneficiary by phone before conducting the interview to determine the patient's eligibility to participate in the study. If a beneficiary had no awareness of being enrolled in CCM services, the interviewer thanked the person, ended the call, and identified the beneficiary as ineligible. Beneficiaries who were deceased or incapacitated with no caregiver to respond on their behalf were also declared ineligible. Beneficiaries who did not recall signing the consent form but were familiar with CCM services were considered eligible to participate in an interview.

We reached 168 beneficiaries by phone. Of those, 45 beneficiaries and 3 caregivers of beneficiaries completed the 
interview. Forty-nine beneficiaries were ineligible and 71 refused to participate.

\section{Coding and Analysis}

With permission from the responding beneficiaries, we recorded the interviews and produced verbatim transcripts for purposes of coding and analysis. Three beneficiaries did not consent to having the interview recorded. In these cases, the interviewer took notes on the beneficiary's responses during the interview and reviewed the notes immediately afterwards to ensure that they were as complete and accurate as possible.

At the start of data collection, we developed a coding rubric with a list of a priori codes based on the semi-structured interview guide. After completing several interviews and reviewing the transcripts, we added some emergent codes to the rubric based on the interview data and created more specific subcategories within some of the a priori codes. ${ }^{12,13}$ Each interviewer coded her own transcript using NVivo11. We met weekly throughout data collection to discuss beneficiary responses and their associated codes. After approximately 40 interviews, the responses started to converge around similar themes. We continued to conduct interviews and discuss responses until we completed 48 interviews, at which point we reached theoretical saturation.

The lead interviewer reviewed each complete transcript and compared it to the coded text for quality control purposes. After reviewing all responses for a given code or theme, the lead researcher developed a descriptive summary that captured the full range of responses, while also distinguishing commonly reported responses from those reported by only a few beneficiaries.

Table 1 Beneficiary Characteristics

\begin{tabular}{ll}
\hline \hline Total number of respondents & $\mathbf{4 8}$ \\
\hline Age & \\
64 and younger & 6 \\
$65-75$ & 24 \\
$76-84$ & 16 \\
85 and over & 2 \\
Sex & 22 \\
Female & 26 \\
Male & \\
Insurance status & 4 \\
Medicare only & 37 \\
Medicare and commercial supplemental & 7 \\
Dual eligible (Medicare and Medicaid) & \\
Number of CCM claims in prior 9 months & 16 \\
2 & 20 \\
3-6 $7-10$ & 12 \\
State of residence & 3 \\
California & 6 \\
Florida & 5 \\
New York & 4 \\
Texas & 30 \\
Other states &
\end{tabular}

\section{RESULTS}

Table 1 presents the distribution of beneficiaries by age, sex, coverage type, location, and number of CCM claims. Thirtytwo of the 48 beneficiaries had 3 or more CCM claims in the prior 9 months.

\section{How Patients Learn About CCM Services and Their Reported First Impressions}

Patients initially learned about CCM services in one of three ways. Most were approached about CCM during an office visit with their primary care physician. A small number of patients reported receiving a letter or a phone call from their practice or from a care manager explaining the service and inviting them to follow up with their physician if they were interested or had questions about participating. Approximately one in five patients did not recall how or when they first learned about CCM services.

More than half of the patients who remembered the initial discussion about CCM reported positive first impressions, citing potential anticipated benefits. For example, several patients liked the idea that they could easily access a member of their care team when they have questions or concerns that may not warrant an office visit. As one patient noted, "It's always comforting to know that someone's there that you can contact." Another patient stated, "I thought it was a pretty good process to stay on top of my health." Other beneficiaries appreciated that their doctor would be compensated for time spent coordinating their care or communicating with them between office visits. One such patient explained, "we bother them a lot with phone calls and emails and it takes their time... my primary care doctor just does not get paid enough."

Other patients who remembered the initial discussion about CCM services expressed varying opinions; some had neutral feelings, others reported some initial concerns, and still others thought CCM services sounded like a good idea for other patients with more serious chronic conditions but were not certain they needed it. Three patients who reported initial concerns questioned the underlying implications of the conversation. One such patient explained, "My first impression, [was], 'Am I really worse than I thought?' Yeah, I was a little apprehensive." Two patients questioned whether the services might feel intrusive. One of these patients, who described herself as "a pretty private person," decided to participate after the provider explained that "it was more or less to benefit all the patients, and that [it] would give them a little bit more quality care."

\section{Patient Perceptions of the Informed Consent Process}

We asked patients about the informed consent process to assess whether any aspect of that process - such as authorizing the clinician to bill for CCM services or to share the patient's electronic medical records with other members of the care 
team-raised any concerns or influenced the way they felt about their provider. Two out of five patients did not remember their provider requesting their consent. Most other patients had little to say about the consent procedures, although a few expressed some initial skepticism about CCM services. As one of these patients described, "When we first started talking about it, it was a matter of, what are you trying to sell me? After I had learned a little bit more about it by reading the form and getting a little bit more clarification, I was comfortable with it."

With the exception of two patients who had withdrawn from CCM by the time of the interview, most patients said the discussion about CCM services had no effect on their relationship with their provider. One beneficiary stated, "I trusted him then, trust him now, and felt like it was just maybe an additional service that could be beneficial." Other patients said their perceptions of their provider improved following the initial discussion about CCM services. One caregiver of a beneficiary explained, "It shows me that he really cares about his patients." Another beneficiary stated he was "impressed that (my doctor) was doing something to stay in touch with me a little more than he had been doing before."

\section{Role of Supplemental Insurance in Beneficiary Decision-making}

Most patients had no out-of-pocket costs for CCM services because the fees were covered by their supplemental insurance or by Medicaid. Many of them did not remember discussing CCM fees or coinsurance with their doctor or said they dismissed consideration of the fees after their doctor explained they would not incur any out-of-pocket costs. Two patients who received a bill for coinsurance for CCM services subsequently revoked the services.

About half of the patients with supplemental insurance were uncertain whether they would have consented to CCM services if they had to pay out-of-pocket. Several patients explained that having supplemental insurance made it easy for them to "at least give it a try." Patients who said they would not have consented if the $20 \%$ coinsurance was not covered offered a few explanations. Some felt CCM services may be beneficial for other patients but did not think they needed it enough to justify additional out-of-pocket costs. Others did not think they could afford additional healthcare costs beyond what they paid for their supplemental insurance.

Three patients who paid the coinsurance out-of-pocket provided similar reasons for feeling undeterred by the cost. One patient particularly valued the monthly calls from his care manager and explained, "You don't really miss the $\$ 12.00$ a month and it's well worth it because you know how busy doctors are now." Similarly, another patient noted, "It's useful to be able to speak to somebody monthly about ongoing problems or something new that has come up." The third patient considered the out-of-pocket cost "minimal for what's involved."

\section{Reasons for Providing Consent}

Patients described several reasons for agreeing to participate in CCM services. Many said they saw no harm in trying it, considering they would not have to pay any out-of-pocket costs. Other patients said they readily gave consent once they learned that "it's something you can stop anytime, if you thought it wasn't a good idea, or if it didn't have any value."

Other patients said they consented to CCM services because the idea "sounded good" when their practitioner introduced it, and they felt it would offer peace of mind. Many of these patients mentioned new or ongoing health problems that had become a growing concern, and they felt they could benefit from more regular communication with their practice. As one patient said, "I felt, at that point, that it was to my advantage for them to be able to coordinate between different doctors for senior care." Another beneficiary said, "It sounded like a good idea to have somebody else in there that you could call and talk to and ask questions and then she would find the answer and get back to us."

Patients also cited trust in their doctor and feeling their doctor should be better compensated as reasons for providing consent for CCM services. Four beneficiaries believed participation was a requirement under Medicare; one of these noted their provider was now requiring CCM participation for particular patients if they wanted to continue to receive care in the practice.

Three beneficiaries had withdrawn their consent for CCM by the time we interviewed them because they did not feel they needed support managing their health conditions.

\section{Care Continuity and Communication with CCM Practice}

In response to questions about continuity of care, patients indicated that they continued to see their usual CCM provider during regular office visits, and the frequency of those visits had not changed in the past year. In addition to those office visits, patients typically received regular phone calls from a care manager, nurse, or their usual clinician.

Several patients reported improved coordination across their care team since they started receiving CCM services. As one patient stated, "Sometimes things that happen to you, where you're seeing a specialist or you're having this checked, sometimes you have a tendency to forget something that was pretty important for [the primary care provider] to know in your care going forward ... this was a way to keep them involved in my total care, whether they were administering it or somebody else was administering it." Some patients appreciated having the same person contact them between appointments to check in and address any ongoing health concerns. One patient explained why this continuity was important to him, saying, "You're talking to the same person every time. It's somebody that knows my history, knows my medications, knows the doctors I'm seeing, knows what I'm being treated for." 


\section{Timely Access to Care}

Many patients felt that participating in CCM services provided them with more timely access to their CCM practice, and they appreciated being able to contact a nurse or care manager who could quickly communicate with the physician or schedule an appointment if indicated. As one beneficiary explained: "I felt like I had an in to the doctor, like there was somebody else to help me through that process [rather] than [my] just calling the main number and then you wait on hold and then they have to type it in the computer and then they have to get it to the doctor."

A small number of beneficiaries who previously had concerns about taking up too much of a doctor's time reported feeling more comfortable calling the office with a question or concern, knowing there was someone available who could respond promptly and could put them in touch with the doctor if the situation merited that kind of attention.

\section{Care Management and Care Transitions}

Patients generally appreciated the monthly phone calls from their provider or a care manager and described the individuals as "reassuring" and "a good reminder." One beneficiary, for example, described how the monthly phone calls helped him and his wife, who was also receiving CCM services, remain mindful of their health: "We think about our health more and what we're doing right or wrong with these phone calls that we're getting every month now. It's a good thing." Another beneficiary described how having more regular communication with his provider influenced him to continue taking his medications despite some undesirable side effects.

Other beneficiaries described additional benefits of the regular check-ins: "They call when it's convenient for me to chat. If I can't immediately, they ask questions. If there's anything I need, boom, I get a quick, rapid response from the doctor's office." Some believed receiving regular calls from the practice spared them unnecessary visits to the office and freed the doctor from spending time on questions or problems that a nurse or care coordinator could address.

About one-quarter of the 48 beneficiaries reported an emergency department visit or overnight hospital stay in the past 3 months, and their reports of follow-up efforts by the practice were mixed. Some beneficiaries said their CCM providers contacted them after they were discharged, others said they initiated contact with the practice, and a few patients did not recall any follow-up from their CCM practice.

\section{DISCUSSION}

We examined chronic care management services from the perspective of participating patients during the first 9 months of CMS launching this new policy.

Patients reported multiple benefits of participating in CCM services, including better access to their primary care team and improved care coordination. With the exception of a few patients who voiced objections to the fee (two of whom had withdrawn from the program), patients reported no downside to participating in CCM services.

When asked how they felt after their doctor explained various aspects of CCM services-such as the cost sharing - many patients had little to say about the discussion. Most patients had supplemental insurance and were consequently unconcerned about cost sharing. A few patients reported some initial skepticism about CCM services but provided consent knowing they could discontinue the service if they were not happy with it.

Some patients felt they were relatively healthy and questioned whether CCM services were warranted in their case. This finding may indicate some "over-recruitment" for CCM services; alternatively, some patients may need coaching on some of the ways they can engage more fully with the services and derive more benefits in the future.

A few study limitations merit consideration. First, as with all qualitative studies, our findings are not generalizable to the entire population of Medicare beneficiaries that participate in CCM services. Additionally, while beneficiaries had to have received at least 2 months of CCM services to be interviewed for our study, some respondents had more months of CCM exposure than others.

To support the adoption of CCM, CMS recently published its changes to the CCM payment policy, which took effect January $1,2017 .{ }^{14}$ The changes include additional billing codes to increase the payment amounts for more complex and time-consuming CCM. CMS also now allows verbal rather than written patient consent as long as it is documented in the patient's record. Patients with supplemental insurance typically paid little attention to the coinsurance amount, so it is difficult to assess how they might respond if that amount increased. The additional billing codes might only apply to a subset of the patients we interviewed, since the patients who considered themselves relatively healthy may not require the same level of CCM as patients with more complex health problems. Though the patients who reported multiple, complicated health problems generally expressed great appreciation for CCM services, it is not clear how they would respond to increased coinsurance amounts, particularly if they had to pay the difference out-of-pocket.

Our findings have implications for providers when approaching patients about CCM services. First, when introducing CCM services, providers may need to tailor the way they frame it, based on their understanding of the patient. Some of the patients we interviewed did not think of themselves as having chronic diseases, and a few reported feeling apprehensive about their health when their provider first talked with them about CCM. With some patients, shifting the focus away from chronic disease management and towards "staying on top of one's health" may make initial conversations about $\mathrm{CCM}$ easier. Also, patients generally felt reassured knowing they can withdraw from CCM any time, suggesting the 
importance of ensuring that patients understand their options for withdrawing consent.

Explaining the aspects of CCM services that patients found empowering, such as having 24/7 access to the CCM team, might also promote more participation. A recent study examining the perceptions and experiences of CCM billing practices found that providers perceived several patient benefits of CCM participation, including improved access to care team members, care continuity, and care coordination. ${ }^{15}$ Providers also reported time management benefits that enabled them to focus more attention on their patients during office visits. Discussion of these benefits-which seem to closely mirror the aspects of CCM that patients found most valuable-is likely to resonate with patients and may encourage their participation.

Corresponding Author: Claire Wilson, $\mathrm{PhD}$; Insight Policy Research, Arlington, VA, USA (e-mail: cwilson@insightpolicyresearch.com).

Funding This work was supported by the Department of Health and Human Services, CMS, under contract HHSM-500-2014-00034I, Task Order No. HHSM-50O-TOOO3.

\section{Compliance with Ethical Standards:}

Conflict of Interest: The authors declare that they do not have a conflict of interest.

\section{REFERENCES}

1. Gerteis J, Izrael D, Deitz D, et al. Multiple chronic conditions chartbook. AHRQ Publications No. 14-0038. Rockville, MD: Agency for Healthcare Research and Quality, U.S. Department of Health and Human Services; 2014.

2. Centers for Medicare \& Medicaid Services. Chronic conditions among Medicare beneficiaries, Chartbook: 2012 edition. Baltimore, MD; 2012.
3. Sharma MA, Cheng N, Moore M, Coffman M, Bazemore AW. Patients with high-cost chronic conditions rely heavily on primary care physicians. J Am Board Fam Med, 2014; 27(1), 11-12.

4. Goodnell S, Bodenheimer T, Berry-Millet R. The Synthesis Project: new insights from research results. Robert Wood Johnson Foundation. Policy Brief No. 19. 2009. Retrieved from http://www.rwjf.org/content/dam/ farm/reports/issue_briefs/2009/rwjf49853. Accessed 1 October 2018.

5. Holahan J, Schoen C, McMorrow S. The potential savings from enhanced chronic care management policies. Washington DC: Urban Institute; 2011.

6. Frandsen BR, Joynt KE, Rebitzer JB, Jha AK. (2015). Care fragmentation, quality, and costs among chronically ill patients. Am J Manag Care. 21(5):355-362.

7. Bleich SN, Sherrod C, Chiang A, et al. Systematic review of programs treating high-need and high-cost people with multiple chronic diseases or disabilities in the United States, 2008-2014. Prev Chronic Dis. 2015;12:150275.

8. Grumbach K, Bodenheimer T, Grundy $\mathbf{P}$. The outcomes of implementing the patient- centered medical home interventions. Washington, DC: Patient-Centered Primary Care Collaborative; 2009.

9. McCall N, Cromwell J, Urato C. Evaluation of Medicare care management for high cost beneficiaries (CMHCB) demonstration: Massachusetts General Hospital and Massachusetts General Physicians Organization (MGH). Research Triangle Park, NC: RTI International; 2010.

10. CMS-1612-FC. "CY 2015 Revisions to Payment Policies Under the Physician Fee Schedule and Other Revisions to Medicare Part B." Nov. 13, 2014. Available at http://www.cms.gov/Medicare/MedicareFee-for-Service-Payment/PhysicianFeeSched/PFS-Federal-RegulationNotices-Items/CMS-1612-FC.html. Accessed 1 October 2018.

11. Schurrer J, O'Malley A, Wilson C, McCall N, Jain N. Evaluation of the diffusion and impact of the chronic care management (CCM) services: final report. Washington DC: Mathematica Policy Research, Inc.:2017.

12. Miles MB, Huberman M. Qualitative data analysis: a methods sourcebook. 3rd edition. Sage Newcastle upon Tyne; 2014.

13. Taylor SJ, Bogdan R. Introduction to qualitative research methods. Hoboken, NJ: Wiley and Sons; 1998.

14. Department of Health and Human Services, Centers for Medicare and Medicaid Services. 80170 Federal Register / Vol. 81, No. 220 / Tuesday, November 15, 2016 / Rules and Regulations. Available at: https://www. gpo.gov/fdsys/pkg/FR-2016-11-15/pdf/2016-26668.pdf. Accessed 1 October 2018

15. O'Malley AS, Sarwar RS, Keith R, Balke P, Ma S, McCall N. Provider experiences with chronic care management (CCM) services and fees: a qualitative research study. J Gen Intern Med. 2017; 32(12):1294-1300. 